\title{
Modulation of staphylokinase- dependent plasminogen activation by mono- and divalent ions
}

\section{A. Yarzábal' \\ R.L. Serrano ${ }^{2}$ \\ and J. Puig ${ }^{1}$}

\author{
${ }^{1}$ Laboratorio de Biologia y Medicina Experimental and \\ ${ }^{2}$ Genetica y Q uimica Celular, Facultad de Ciencias, Universidad de Los Andes, \\ Merida, Venezuela
}

\begin{abstract}
\section{Correspondence}

A. Yarzábal

LABIO M EX, Facultad de Ciencias

Universidad de Los Andes

Apartado Postal 281

Mérida 5101

Venezuela

Fax: + 58-74-63-4587

E-mail: yluis@ing.ula.ve

Research supported by CDCHT/ ULA (No. C-678-94).

The effect of several ions $\left(\mathrm{Cl}^{-}, \mathrm{Na}^{+}, \mathrm{K}^{+}, \mathrm{Ca}^{2+}\right)$ on the rate of plasminogen $(\mathrm{Pg})$ activation by recombinant staphylokinase (rSTA) is reported. Both monovalent and divalent ions affect the rate at which $\mathrm{Pg}$ is activated by rSTA, in a concentration-dependent manner (range 0-100 $\mathrm{mM}$ ). In almost all cases, a decrease of the initial velocity of activation was observed. $\mathrm{Cl}^{-}$showed the most striking inhibitory effect at low concentrations $(64 \%$ at $10 \mathrm{mM})$. However, in the presence of a fibrin surface, this inhibition was attenuated to $38 \%$. Surprisingly, $10 \mathrm{mM}$ $\mathrm{Ca}^{2+}$ enhanced the Pg activation rate $21 \%$ when a polymerized fibrin matrix was present. These data support the idea that ions can modulate the rate of $\mathrm{Pg}$ activation through a mechanism that may be associated with changes in the molecular conformation of the zymogen. This effect is strongly dependent on the presence of a fibrin clot.
\end{abstract}

Received March 17, 1998

Accepted October 7, 1998

\section{Key words}

- Staphylokinase

- Plasminogen activation

- Fibrinolysis
Staphylokinase (STA), a bacterial plasminogen activator produced by some strains of Staphylococcus aureus, is considered at present as the most promising fibrinolytic agent for the treatment of patients with acute myocardial infarction (1). Human plasminogen $(\mathrm{Pg})$ activation by STA is being studied to elucidate the mechanism of activation since STA is not an enzyme. It has been established that STA forms a stoichiometric complex with Pg (STA.Pg) in which a plasmin $(\mathrm{Pm})$ activity is rapidly detected. The STA.Pm complex is the active enzyme which specifically activates other Pg molecules on the surface of a fibrin clot $(2,3)$. This preferential activation of fibrin-bound $\mathrm{Pg}$ has been explained by the rapid inhibition of circulating complexes by $\alpha$-2-antiplasmin in plasma but not on the fibrin surface, where the molecular domains involved in the inhibition process closely associate with the clot (4).

Kinetic studies of Pg activation by STA have focused mainly upon the activation mechanism, fibrin specificity, the correlation among various inhibitors and the effect of STA concentration on plasminogen activation (5). It is well known that ions could act as negative or positive effectors of Pg activation by streptokinase and urokinase, two wellknown Pg activators currently in therapeutic use $(6,7)$. However, to date no results concerning these effects on Pg activation by STA have been described. We report here, for the first time, the effect of $\mathrm{Ca}^{2+}$ and $\mathrm{Cl}^{-}$, as well as other ions, on the rate of Pg activation by recombinant STA (rSTA). 
rSTA and human Glu-Pg were purified and characterized as described elsewhere $(8,9)$. Lys-Pg was produced by plasmin digestion of Glu-Pg at $37^{\circ} \mathrm{C}$ in $40 \mathrm{mM} \mathrm{L-lysine}$ for $1 \mathrm{~h}$ (10). rSTA.Pm complexes were obtained by incubation of Pg with $\mathrm{rSTA}(3 \mu \mathrm{M}$ each, with a $5 \%$ molar excess of rSTA) at $37^{\circ} \mathrm{C}$ for $10 \mathrm{~min}$ in $50 \mathrm{mM}$ HEPES, pH 7.4, containing $25 \%$ glycerol (11). Solid phase fibrin plates were prepared to activate plasminogen in the presence of a polymerized fibrin surface. Briefly, after treatment of polyvinyl chloride (PVC) plates with $2.5 \%$ glutaraldehyde, purified human fibrinogen $(0.1$ $\mathrm{mg} / \mathrm{ml}$ in $0.1 \mathrm{M}$ sodium phosphate buffer, $\mathrm{pH}$ 7.4, containing $1 \mathrm{mM} \mathrm{CaCl}_{2}$ ) was covalently fixed and converted into a fibrin network by thrombin treatment (12). Activation assays were performed in microtiter and fibrin plates and were based on the development of amidolytic activity of Pm generated by $\mathrm{Pg}$ activation, towards a chromogenic

Figure 1 - Kinetics of plasminogen activation by rSTA at $37^{\circ} \mathrm{C}$. Glu-Pg (A) and Lys-Pg (B) at different concentrations were activated with $50 \mathrm{nM}$ rSTA in the presence of a chromogenic substrate (CBS 00.65). Absorbance at $405 \mathrm{~nm}$ was monitored every minute and $\mathrm{dA} / \mathrm{dt}$ was plotted against time. Squares $(4.5 \mu \mathrm{M})$, triangles $(1.7 \mu \mathrm{M})$, circles $(0.85$ $\mu \mathrm{M})$. C, Double reciprocal plot of the rate of plasminogen activation by rSTA, as a function of plasminogen concentration. GluPg (squares), Lys-Pg (circles).
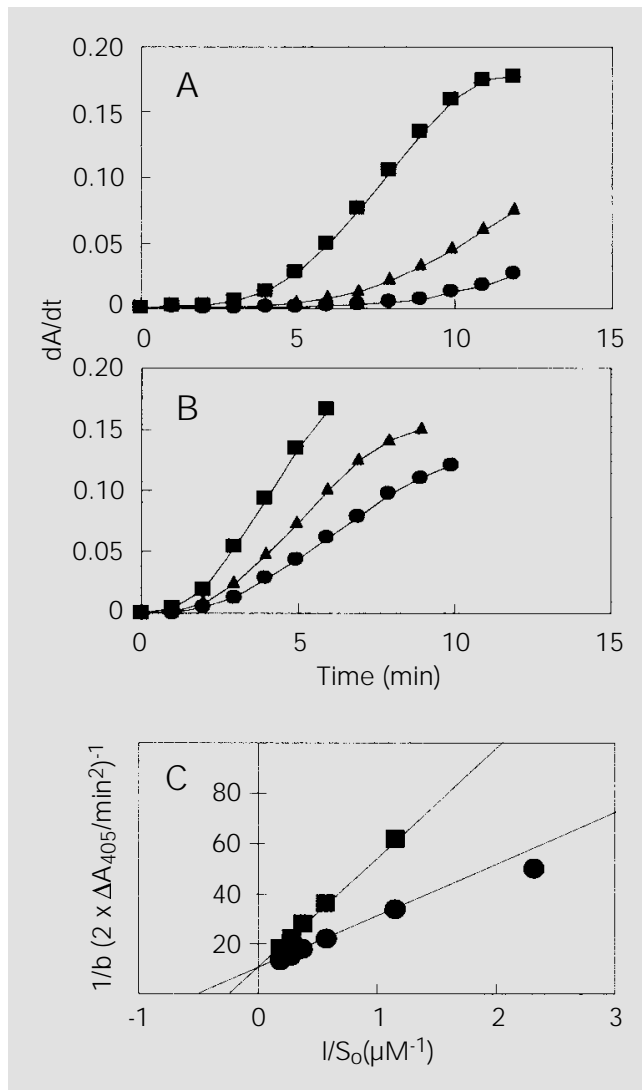

substrate specific for plasmin, MM-Hyp-ArgpNA (CBS 00.65, Diagnostica STAGO, Asnieres, France) (13). The assays were performed at $37^{\circ} \mathrm{C}$ in a buffer containing $50 \mathrm{mM}$ HEPES, pH 7.4, the substrate CBS 00.65 (1 $\mathrm{mM})$, rSTA $(50 \mathrm{nM})$ or rSTA.Pm complexes $(10 \mathrm{nM})$ and different levels of ions ranging from 0 to $100 \mathrm{mM}$. Glu-Pg $(1 \mu \mathrm{M})$ was added to start the reaction and the absorbance at $405 \mathrm{~nm}$ of the mixtures was continuously monitored. Initial reaction rates were determined from the slopes of plots of $A_{405}$ versus $t^{2}$, according to the method of Wohl (14).

As expected, rSTA activated human GluPg with a lag period of at least 5 min which almost disappeared when Lys-Pg was used (Figure 1A and B). Activation of Pg by rSTA obeyed Michaelis-Menten kinetics as revealed by linear double-reciprocal plots of the initial activation rates versus the Pg concentration (Figure 1C). When preformed rSTA.Pm complexes were used as the activator species, the lag period also diminished. This was also observed when activation assays were performed in the presence of a fibrin surface on fibrin plates (results not shown).

Taken together, these results support the view that during the activation process of Glu-Pg by STA there is a rate-limiting step during which the activator must bind to the zymogen causing a molecular conversion of Glu-Pg to Lys-Pg to take place. It has been shown that Lys-Pg possesses a more open conformation and interacts more easily with activators (15). In fibrin plates, the binding of Glu-Pg to fibrin through kringle domains induces a similar conformational change, from a "closed" to an "open" form that accelerates the activation rate (16).

These observations were confirmed when the activation experiments were carried out in the presence of different ions (Figure 2). The effect of $\mathrm{Cl}^{-}$, which stabilizes the "closed" form of the zymogen (17), is markedly inhibitory even at $1 / 10$ of its physiological concentration. SDS-PAGE analysis of 


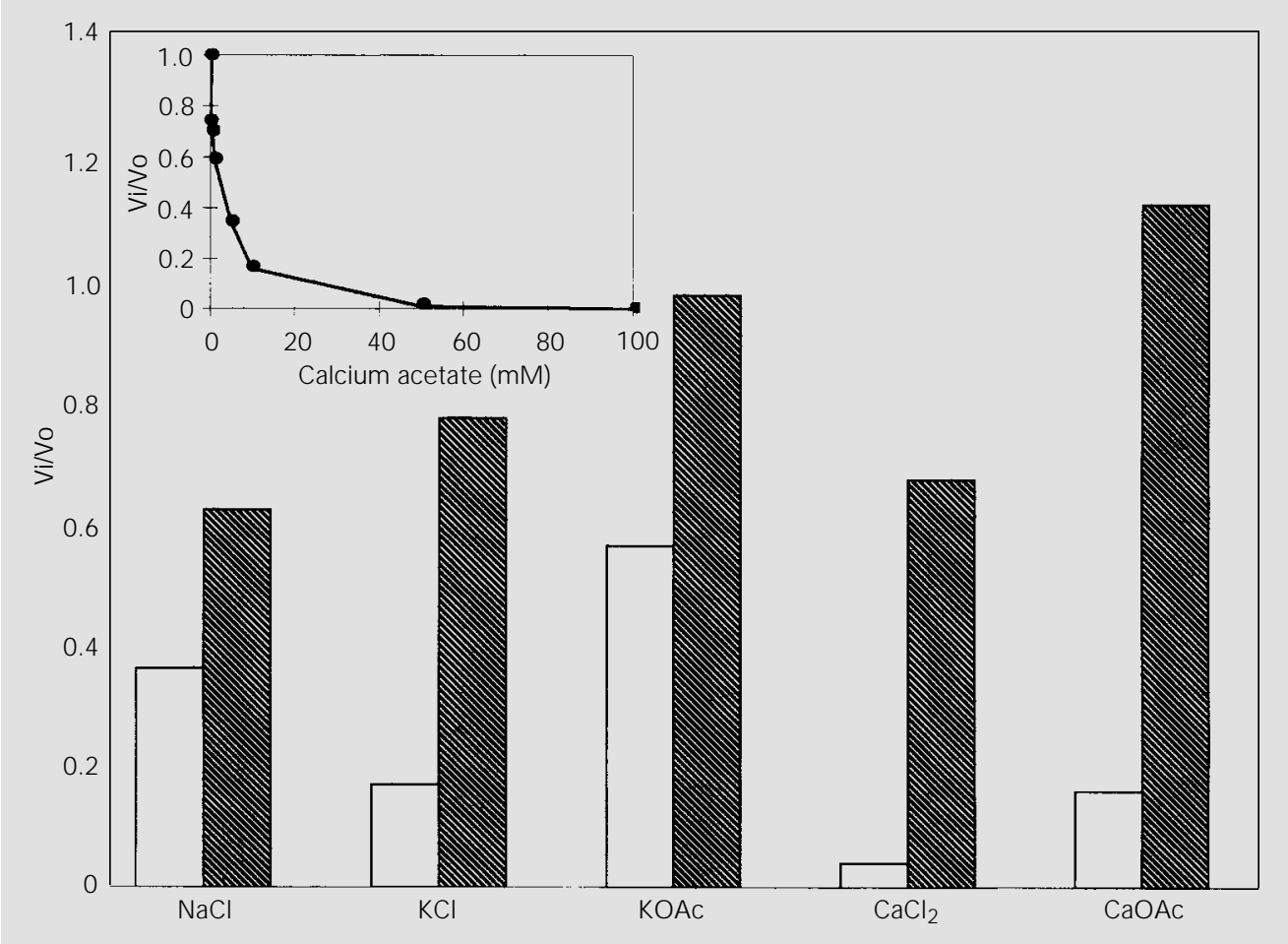

Pg activation products revealed that in the presence of $20 \mathrm{mM} \mathrm{NaCl}$ the one-chain Glu$\mathrm{Pg}$ form did not undergo the molecular transition to the enzymatically active two-chain $\mathrm{Pm}$ (not shown). $\mathrm{Cl}^{-}$-induced inhibition has been reported previously in studies where $\mathrm{Pg}$ was activated with streptokinase and urokinase, two fibrinolytic agents used worldwide for the treatment of acute myocardial infarction (5). However, in the presence of a fibrin surface, a significant reduction of the inhibitory effect of $\mathrm{Cl}^{-}$was observed. Once again, Pg binding to fibrin appears to reverse the inhibition, probably due to $\mathrm{Cl}^{-}$exclusion from the domains involved in such interaction (17).

The inhibitory effect of other mono- and divalent ions has also been established. Their effect was not so striking as the $\mathrm{Cl}^{-}$inhibition, but a concentration-dependent decrease of the Pg activation rate was observed in almost all cases (Figure 2, inset). The anion acetate does not inhibit Glu-Pg activation to the same extent as $\mathrm{Cl}^{-}$. As already stated, acetate does not bind as strongly to the anion-binding sites on Glu-Pg kringles as does $\mathrm{Cl}^{-}$and therefore does not modulate as effectively the production of the "closed" state (17). Surprisingly, when calcium in the acetate salt form was added to activation mixtures in fibrin plates, an enhancement of the rate of Pg activation by rSTA was observed. This effect attained its maximum at a concentration of $5 \mathrm{mM}$, which is close to the physiological concentration.

The role of $\mathrm{Ca}^{2+}$ in fibrinolysis is unknown and has been investigated by several authors. Stack et al. (7) observed that divalent cations inhibited the activation of GluPg but stimulated the activation of Lys-Pg, confirming that the molecular conformation of the substrate is critical for the inhibitory/ enhancer effect. Markus et al. (18) observed that casein, a $\mathrm{Ca}^{2+}{ }^{2}$-rich phosphoprotein, is a potent accelerator of the rate of $\mathrm{Pg}$ activation by both urokinase and tissue Pg-activator. Their findings showed that partial removal of $\mathrm{Ca}^{2+}$ by chelating agents such as EDTA or
Figure 2 - Effect of ions on the rate of plasminogen activation by rSTA. Pg was activated by rSTA in 50 mM HEPES, pH 7.4, in the presence of a chromogenic substrate for plasmin (MM-Hyp-Arg-pNA, CBS 00.65) and different ions (10 $\mathrm{mM}$ each). $\mathrm{Vi}$ and $\mathrm{Vo}$ are the initial velocities of hydrolysis of CBS 00.65 by plasmin in the presence and absence of ions, respectively. Data are reported as the mean of three experiments. Experiments were performed on PVC plates in the absence (open bars) and in the presence of a fibrin surface (striped bars). Inset, Concentration-dependent decrease of the initial plasminogen activation rate in the presence of increasing concentrations of calcium acetate and in the absence of a fibrin surface. 
EGTA reduced the enhancing effect by an average of $38 \%$ of the control. Recently, Kojima et al. (19) reported that a physiological concentration of $\mathrm{Ca}^{2+}$ significantly shortened the euglobulin clot lysis time, an assay used to assess systemic fibrinolytic activity. Furthermore, tetranectin, a plasminogenbinding protein which possesses intact calcium binding sites, has been shown to interact specifically with kringle 4 . The function of tetranectin is unknown, but the protein has been reported to enhance Pg activation by t-PA, a mechanism that could be closely associated with calcium binding (20).

Our findings add to these results, showing that $\mathrm{Ca}^{2+}$ enhances the rate at which Pg is activated by rSTA in the presence of polymerized fibrin. Even though the role of $\mathrm{Ca}^{2+}$ requires further investigation, it is clear that $\mathrm{Ca}^{2+}$ may modulate the rate at which $\mathrm{Pg}$ is converted to Pm by physiological and therapeutical activators. Such modulation of fibrinolytic activity should be very important in determining the rate of activation in the microenvironment of this zymogen, particularly in the vicinity of a fibrin clot.

Nevertheless, since the mechanism of Pg activation is triggered and further amplified by specific interactions of fibrin with the $\mathrm{Pg}$ kringle domains, it is clear that these studies should be developed at least in the presence of a fibrin surface in order to obtain experimental conditions as close as possible to the physiological plasma/fibrin interphase. If not, some effects could be over- or underestimated, as shown in the present study.

\section{References}

1. Lijnen HR \& Collen D (1996). Staphylokinase, a fibrin-specific bacterial plasminogen activator. Fibrinolysis, 10: 119-126.

2. Collen $D$, Schlott $B$, Engelborghs $Y$, Van Hoef B, Hartmann M, Lijnen HR \& Behnke $D$ (1993). On the mechanism of the activation of human plasminogen by recombinant staphylokinase. J ournal of Biological Chemistry, 268: 8284-8289.

3. Lijnen HR, Van Hoef B, De Cock F, Okada K, Ueshima S, Matsuo O \& Collen D (1991). On the mechanism of fibrin-specific plasminogen activation by staphylokinase. J ournal of Biological Chemistry, 266: 11826-11832.

4. Silence K, Collen D \& Lijnen HR (1993). Interaction between staphylokinase, plasmin(ogen) and $\alpha_{2}$-antiplasmin. Recycling of staphylokinase after neutralization of the plasmin-staphylokinase complex by $\alpha_{2}$-antiplasmin. J ournal of Biological Chemistry, 268: 9811-9816.

5. Shibata H, Nagaoka M, Sakai M, Sawada H, Watanabe T \& Yokokura T (1994). Kinetic studies on the plasminogen activation by the staphylokinase-plasmin complex. J ournal of Biochemistry, 115: 738-
742.

6. Urano $T$, Chibber BAK \& Castellino FJ (1987). The reciprocal effects of $\varepsilon$ aminohexanoic acid and chloride ion on the activation of human (Glu1) plasminogen by human urokinase. Proceedings of the National Academy of Sciences, USA, 84: 4031-4036.

7. Stack S, Gonzáles-Gronow M \& Pizzo S (1991). The effect of divalent cations on the conformation and function of human plasminogen. Archives of Biochemistry and Biophysics, 284: 58-62.

8. Yarzábal A, Bastidas M, Avilán L, CruzJ \& Puig J (1997). Induction conditions for maximizing recombinant staphylokinase expression in Escherichia coli. Biotechnology Letters, 19: 633-637.

9. Castellino FJ \& Powell J R (1981). Human plasminogen. Methods in Enzymology, 80: 365-378.

10. Trieu T, Behnke D, Gerlach D \& Tang J (1993). Activation of human plasminogen by recombinant staphylokinase. Methods in Enzymology, 223: 156-167.

11. Lijnen HR, Van Hoef B \& Collen D (1993). Interaction of staphylokinase with differ- ent molecular forms of plasminogen. European J ournal of Biochemistry, 211: 9197.

12. Anglés-Cano E (1985). A spectrophotometric solid-phase fibrin-tissue plasminogen activator activity assay (SOFIA-tPA) for high-fibrin-affinity tissue plasminogen activators. Analytical Biochemistry, 153: 201-210.

13. Chibber BAK, Morris J P \& Castellino FJ (1985). Effects of human fibrinogen and its cleavage products on activation of human plasminogen by streptokinase. Biochemistry, 24: 3429-3434.

14. Wohl RC, Summaria $L \&$ Robbins KC (1980). Kinetics of activation of human plasminogen by different activator species at pH 7.4 and $37^{\circ} \mathrm{C}$. J ournal of Biological Chemistry, 255: 2005-2013.

15. Mangel WF, Lin $B \&$ Ramakrishan $V$ (1990). Characterization of an extremely large, ligand-induced conformational change in plasminogen. Science, 248: 6973.

16. Ponting CP, Marshall J $M \&$ \& CederholmWilliams SA (1992). Plasminogen: a structural review. Blood Coagulation and $\mathrm{Fi}-$ 
brinolysis, 3: 605-614.

17. McCance SG \& Castellino FJ (1995). Contributions of individual kringle domains towards maintenance of the chloride-induced tight conformation of human glutamic acid-1 plasminogen. Biochemistry, 34: 9581-9586.

18. Markus G, Hitt S, Harvey SR \& Tritsch GL
(1993). Casein, a powerful enhancer of the rate of plasminogen activation. Fibrinolysis, 7: 229-236.

19. Kojima Y, Urano T, Kojima K, Serizawa K, Takada Y \& Takada A (1994). The significant enhancement of fibrinolysis by calcium ion in a cell free system: the shortening of euglobulin clot lysis time by cal- cium ion. Thrombosis and Haemostasis, 72: 113-118.

20. Clemmensen I, Petersen LC \& Kluft C (1986). Purification and characterization of a novel, oligomeric, plasminogen kringle 4 binding protein from human plasma: tetranectin. European J ournal of Biochemistry, 156: 327-333. 